UCRL-JC-129000

PREPRINT

\title{
Supernova Experiments on the Nova Laser
}

\author{
J. Kane, D. Arnett, B. A. Remington, \\ S. G. Glendinning, R. Wallace, \\ A. Rubenchik, and B. A. Fryxell
}

This paper was prepared for and presented at the

Second Oakridge Symposium on Atomic and Nuclear Astrophysics

Oakridge, Tennessee

December 2-6, 1997

December 2, 1997

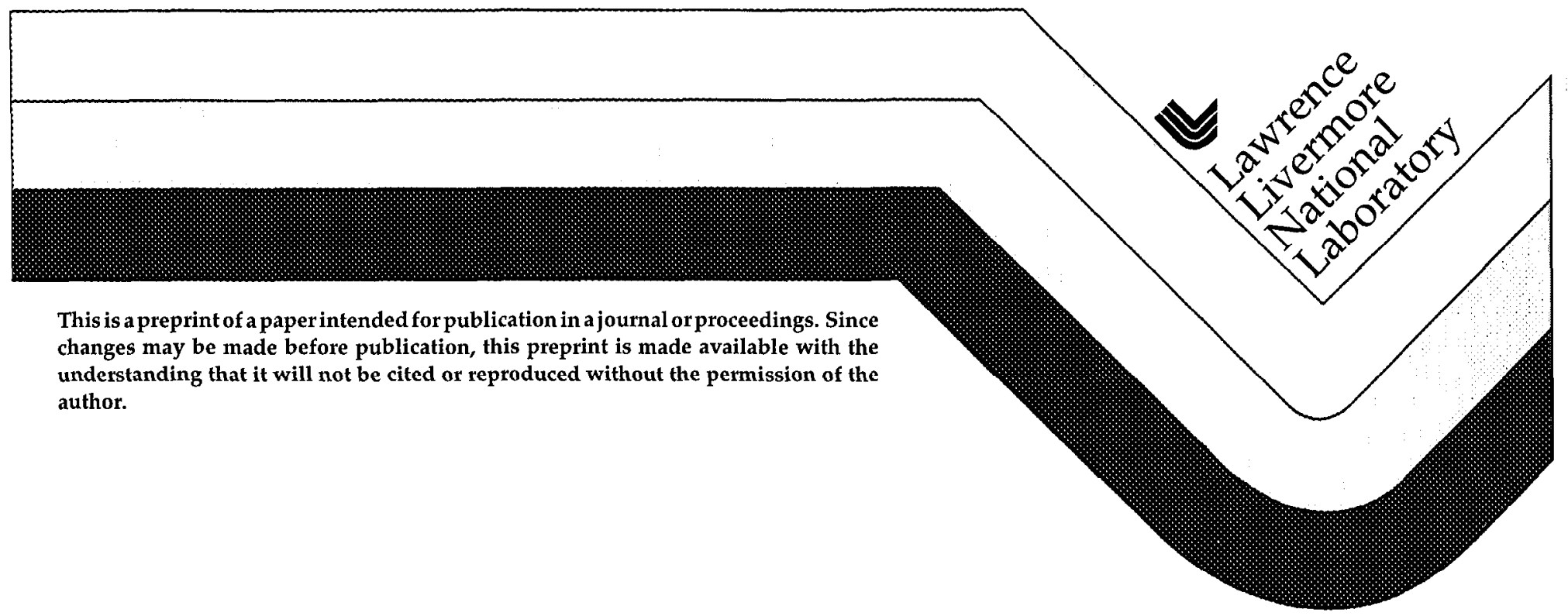




\section{DISCLAIMER}

This document was prepared as an account of work sponsored by an agency of the United States Government. Neither the United States Government nor the University of California nor any of their employees, makes any warranty, express or implied, or assumes any legal liability or responsibility for the accuracy, completeness, or usefulness of any information, apparatus, product, or process disclosed, or represents that its use would not infringe privately owned rights. Reference herein to any specific commercial product, process, or service by trade name, trademark, manufacturer, or otherwise, does not necessarily constitute or imply its endorsement, recommendation, or favoring by the United States Government or the University of California. The views and opinions of authors expressed herein do not necessarily state or reflect those of the United States Government or the University of California, and shall not be used for advertising or product endorsement purposes. 


\title{
Supernova experiments on the Nova laser
}

\author{
J. Kane $\ddagger^{+}$, D. Arnett $\dagger$, B. A. Remington $\neq$, S. G. Glendinning $\ddagger$, \\ R. Wallace $\ddagger$, A. Rubenchik§, B. A. Fryxell \\ † University of Arizona, Tucson, AZ 85721 \\ $\ddagger$ Lawrence Livermore National Laboratory (LLNL), Livermore CA 94550 \\ $\S$ University of California Davis, \| NASA/GFC
}

\begin{abstract}
Supernova (SN) 1987A focused attention on the critical role of hydrodynamic instabilities in the evolution of supernovae. To test the modeling of these instabilities, we are developing laboratory experiments of hydrodynamic mixing under conditions relevant to supernovae. Initial results were reported in [1]. The Nova laser is used to generate a 10-15 Mbar shock at the interface of a two-layer planar target, which triggers perturbation growth, due to the Richtmyer-Meshkov and Rayleigh-Taylor instabilities as the interface decelerates. This resembles the hydrodynamics of the He-H interface of a Type II supernova at intermediate times, up to a few $\times 10^{3} \mathrm{~s}$. The experiment is modeled using the hydrodynamics codes HYADES and CALE, and the supernova code PROMETHEUS. Results of the experiments and simulations are presented. New analysis of the bubble velocity is presented, as well as a study of $2 \mathrm{D}$ vs. $3 \mathrm{D}$ difference in growth at the He-H interface of SN 1987A.
\end{abstract}

\section{Introduction}

Observations of SN1987A, a core collapse supernova (SN) in the Large Magellanic Cloud, strongly suggested the occurence of material mixing driven by the Richtmyer-Meshkov (RM) and Rayleigh-Taylor (RT) instabilities [25, 21, 24, 29, 5]. The 'Bochum event' [28, 15, 8], and early detection of fadioactive ${ }^{56} \mathrm{Co}$ from the explosively burned oxygen layer implied that the ${ }^{56} \mathrm{Co}$ had been mixed well into the outer layers. Doppler broadening of the gamma-ray and optical lines from ${ }^{56} \mathrm{Co}$ implied velocities in excess of $3000 \mathrm{~km} / \mathrm{s}[31,30,20]$, whereas $2 \mathrm{D}$ modeling to date predicts maximum velocities of $\leq 2000 \mathrm{~km} / \mathrm{s}$, suggesting that perhaps $3 \mathrm{D}$ hydro effects should be considered. Given the fundamental role played by the RM and RT instabilities in SN evolution, it is desirable to develop the means of testing the hydrodynamics of the SN codes. We report here on experiments using the Nova laser at Lawrence Livermore National Laboratory (LLNL) to test the modeling of compressible RT instabilities at relevant pressures. We use the SN code PROMETHEUS to model the experiment, and for comparison, the LLNL code CALE. We also present an analysis of the hydrodynamic growth in the experiment in terms of theory for incompressible hydrodynamic instabilities, and report on numerical investigations of $2 \mathrm{D}$ vs. $3 \mathrm{D}$ hydro differences in SN $1987 \mathrm{~A}$.

${ }^{1}$ iave@llnl gov 


\section{1D Simulations of SN and Laser Experiment}

Fig. 1 shows a $20 \mathrm{M}_{\odot}$ model for the progenitor of SN 1987A [4], and from 1D PROMETHEUS (a multi-D Piecewise Parabolic Method [PPM] hydro code), the vclocity profile of the He$\mathrm{H}$ interface induced by the blast wave and subsequent deceleration of the interface, and the 1D pressure and densily profiles al $2000 \mathrm{~s}$. For more details of the SN 1987A explosion, the PROMETHEUS code, and the simulations shown here, see $[5,6,13,22,32,1]$. At the He-H interface, the strong acceleration followed by deceleration, along with the crossed pressure and density gradients suggest that the He-H interface should exhibit strong RM and RT instability, with the full multi-D interface evolving well into the nonlinear regime, as seen in $[6,13,22]$.
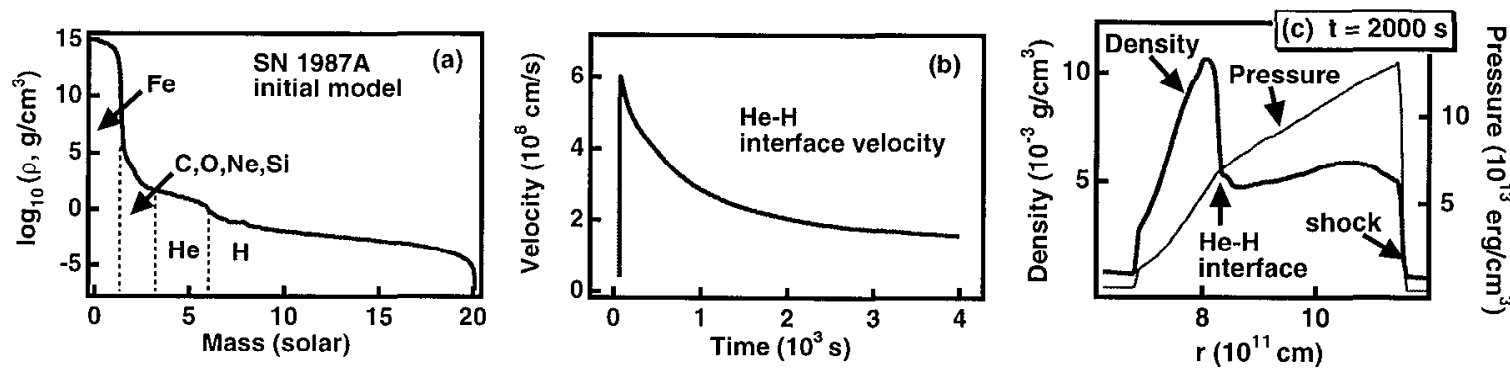

Figure 1. (a) Initial model for SN 1987A (b) He-H interface velocity; note acceleration due to shock, and subsequent deceleration (c) crossed density and pressure gradients at the He-H interface after passage of the shock.
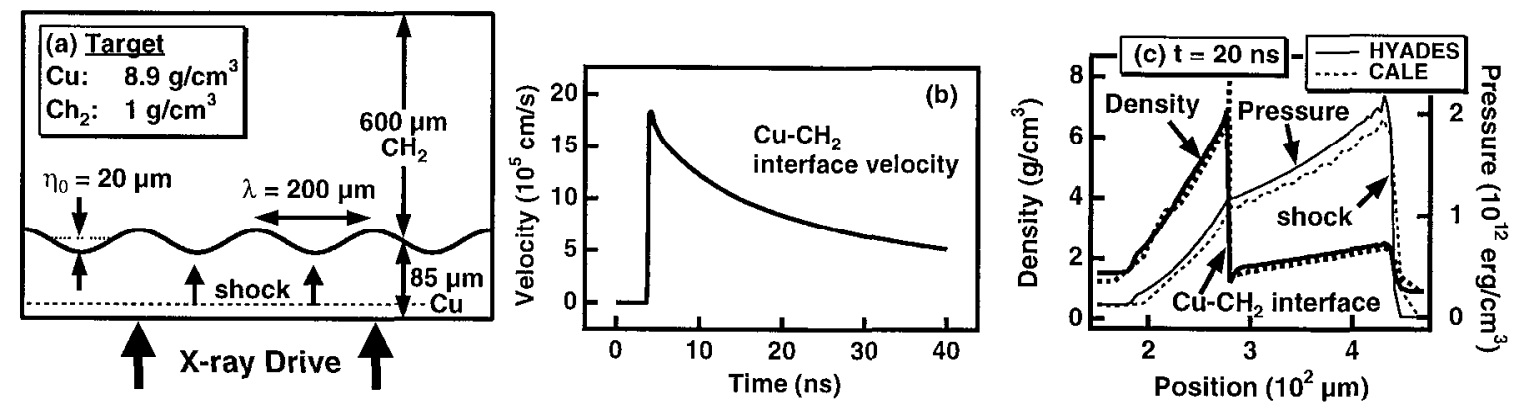

Figure 2. (a) Initial target for Nova experiment (b) $\mathrm{Cu}-\mathrm{CH}_{2}$ interface velocity (c) crossed density and pressure gradients at the $\mathrm{Cu}-\mathrm{CH}_{2}$ interface.

The Nova experimental configuration is illustrated in Fig. 2; for further discussion of the experiment, the experimental techniques, and the codes used, see $[1,23,12,9,19,17,10$, $18,7]$. The Nova laser is used to produce an $\mathrm{X}$-ray drive which shocks a two-layer $\mathrm{Cu}-\mathrm{CH}_{2}$ planar target having an imposed material perturbation at the $\mathrm{Cu}-\mathrm{CH}_{2}$ interface. Figs. $2 \mathrm{~b}$ and c show HYADES simulations of a 1D (unperturbed) experiment; as in the SN case (Fig. 1), the interface is first accelerated by the shock and then decelerated, and the pressure and density gradients are crossed at the interface; the $\mathrm{Cu}-\mathrm{CH}_{2}$ should also exhibit strong $\mathrm{RM}$ and RT instability. We typically observe the experiment for up to $40 \mathrm{~ns}$.

We model the laser experiment using a combination of codes: HYADES, CALE and PROMETHEUS. The HYADES code [18] is a $1 \mathrm{D}$ Lagrangian code with multigroup radiation transport and tabular EOS, and CALE is a 2D Arbitrary Lagrangian Eulerian (ALE) code [7] with tabular EOS and interface tracking. PROMETHEUS was described above. Ideal gas EOS is used for all the PROMETHEUS simulations of the laser experiments. We use the measured radiation temperature, $\mathrm{T}_{r}(t)$, as the energy input to HYADES, and the versions of CALE and 
PROMETHEUS that we are using do not have radiation transport. We begin all simulations using HYADES, then map to PROMETHEUS and CALE at, $2.45 \mathrm{~ns}$, just prior to the arrival of the shock at the thinnest part of the (perturbed) Cu. As seen in Fig. 2c, CALE and HYADES agree well long after the mapping, indicating that the experiment is hydro-dominated. For a discussion of scaling of hydro instability growth between the SN and Nova case, see [1, 2, 11].

\section{Results and 2D Simulations}

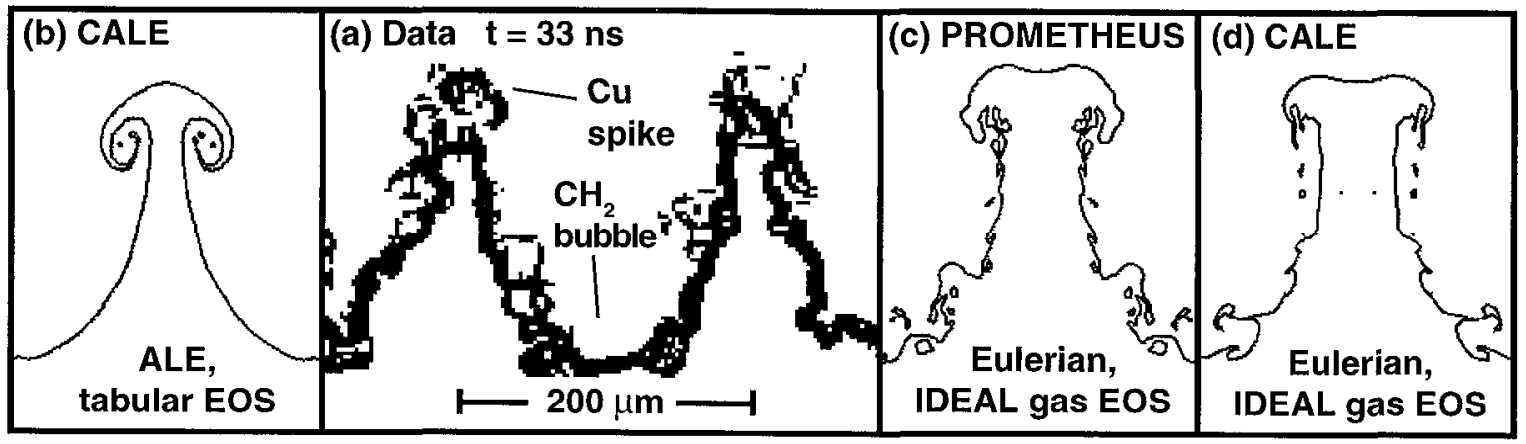

Figure 3. Data and simulations for 2D experiment. (a) Outline of $\mathrm{Cu}$ spikes extracted from radiograph of experiment at $33 \mathrm{~ns}$. The $\mathrm{Cu}$ spikes and $\mathrm{CH}_{2}$ bubbles are clearly evident. (b) CALE simulation, in Arbitrary Lagrangian Eulerian (ALE) mode and using tabular EOS. (c) PROMETHEUS simulation, with fixed (Eulerian) orthogonal grid and ideal gas EOS. There is more fine structure with PROMETHEUS. (d) When CALE is run with Eulerian orthogonal grid and ideal gas EOS, the result is similar to PROMETHEUS, with the fine structure somewhat supressed by the interface tracking in CALE. CALE can be started with an orthogonal or non-orthogonal grid; in the former case the inilial sinusoidal interface must be stairstepped along the grid, while in the latter case the interface can be piecewise smooth. When CALE is run in 8 different ways, (initial interface:smooth/stairstepped) $\times$ (grid:Eulerian[fixed]/ALE) $\times$ (EOS:tabular/ideal), the dominant factor in producing fine structure appears to be the stairstepping (not shown.)
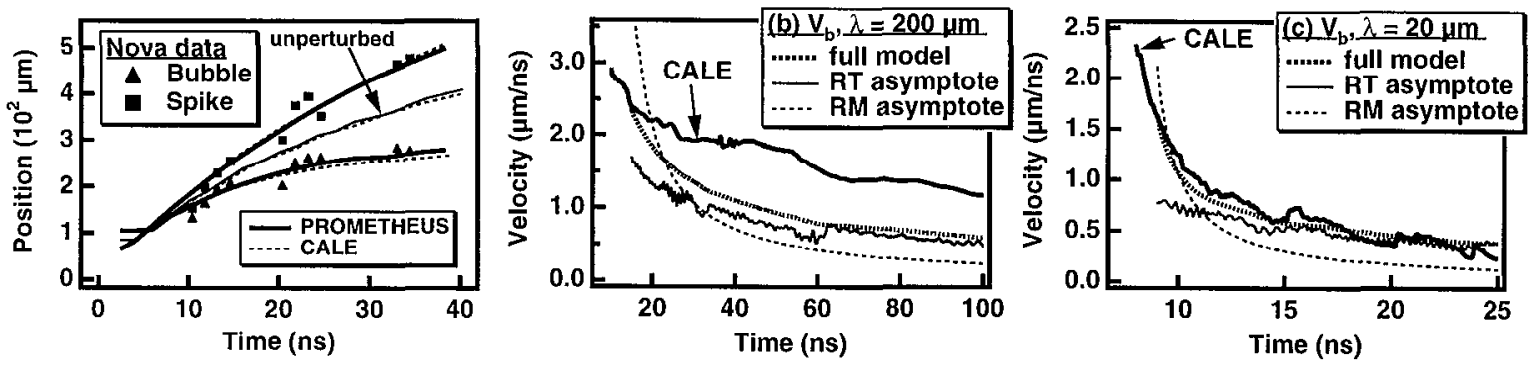

Figure 4. (a) Bubble and spike velocities (b) incompressible growth model vs 'undecompressed' CALE simulation with single mode velocity perturbation, $\lambda=200 \mu \mathrm{m}$ (c) same for $\lambda=20 \mu \mathrm{m}$

Fig. 3a shows a 2D image from the experiment at $t=33.2$ ns. The edge of the $\mathrm{Cu}$ foil shows the classic nonlinear RM/RT bubble-and-spike shape, and there are faint indications of a roll-up at the very tip of the spike. We initiate our $2 \mathrm{D}$ simulations in the same manner as in 1D: we map the conditions from the HYADES calculation at $2.45 \mathrm{~ns}$. Fig. 3b-d show PROMETHEUS and CALE simulations of the experiment. The gross features of the experiment are well reproduced by both simulations. For further details about the data and simulations, see [1]. Fig. 4 compares spike and bubble position from the experiment, CALE, 
and PROMETHEUS. The observed spike and bubble fronts are well reproduced by both hydrodynamics codes. Also shown is the position of an unperturbed interface as calculated by CALE and PROMETHEUS. In [1] we presented a preliminary analysis of the hydrodynamic growth in terms of nonlinear RM and RT theory for semi-infinite fluids [3], and are preparing more detailed analyses for an upcoming publication. In the more detailed analyses we consider the effects of the 'accordionlike' decompression of the $\mathrm{Cu}$ and $\mathrm{CH}_{2}$ layers, the effects of the time varying effective $g$ (interface deceleration,) and the finite thickness of the dense part of the $\mathrm{Cu}$ layer (see. Fig. 2b.) In one of our analyses, we do a $2 \mathrm{D}$ simulation in which we impose a curl-free single mode velocity perturbation at a flat $\mathrm{Cu}-\mathrm{CH}_{2}$ interface, with the same wavelength as the perturbation in the experiment, after the shock passes the interface. We use the results of a 1D simulation to estimate the background decompression velocities eveywhere in the two layers, and then subtract these velocities from the bubble and spike velocities. We then use a drag-vs.-buoyancy model 3 to analyze the 'undecompressed' bubble and spike velocities. In Fig. $4 \mathrm{~b}$ and $\mathrm{c}$, we compare the $2 \mathrm{D}$ bubble velocity $v_{b}$ to the the model, and also to the the instantaneous asynuptolic RT (which accounts for $g$ ) and RM (which assumes $g=0$ after the initial impulsive accleration) velocities (see 3). For the actual wavelength used in the experiment, $\lambda=200 \mu \mathrm{m}$, (Fig. $4 \mathrm{~b}$ ) we find that $v_{b}$ does not agree well with the model, and the model does not approach either the RT or RM asymptote until the growth has become very nonlinear (which occurs by $60 \mathrm{~ns}$.) For a smaller wavelength, $\lambda=20 \mu \mathrm{m}$ (Fig. $4 \mathrm{c}$ ), we find that the $v_{b}$ agrees well with the model, which quickly approaches the RT asymptote. This suggests that the a large rising $\mathrm{CH}_{2}$ bubble is able to push more easily through a dense Cu shell that is relatively thin $(\sim 35-70 \mu \mathrm{m})$ compared to the bubble dimensions $(\lambda)$. Analysis of the spike velocities (not shown) suggest that the spike velocity is much harder to characterize as RMor RT-like, possibly because of the Kelvin-Ilelmholtz instability at the spike tip, and because of the rapidly falling $g$, which drops as $\sim 1 / t$. However to the extent that the hydrodynamics is driven by the rising bubbles, for short wavelengths the growth seems to be RT-like. At the same time, it is clear that many complicating factors make simple classification of the growth difficult; the same is likely to be true for the SN case. We will present more details of this and other analyses in our upcoming paper.

\section{4. $2 \mathrm{D}$ vs. $3 \mathrm{D}$ hydro}
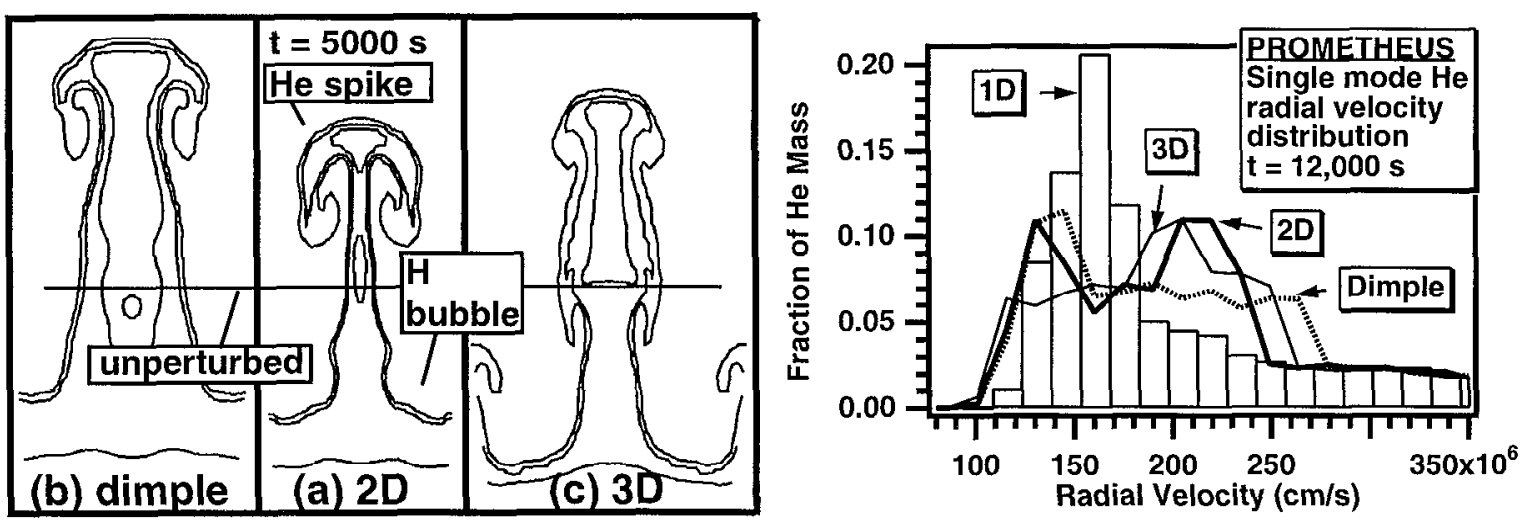

Figure 5. (a,b,c) He-H interface growth for 2D, dimple and 3D perturbations. (d) He velocity distributions.

We are beginning experimental investigations of $2 \mathrm{D}$ vs. $3 \mathrm{D}$ SN-relevant hydro in Nova experiments. 2D vs. 3D differences could help explain why $2 \mathrm{D}$ simulations of SN 1987A 
underpredict the observed ${ }^{56} \mathrm{Co}$ velocities. By drag-vs.-buoyancy arguments [3, 19], a 3D perturbation is expected to grow faster in the nonlinear regime than a $2 \mathrm{D}$ perturbation of the same wave number; perturbation of the same wave number and amplitude grow at the same rate in the lincar regime $[2,3,19]$. We arc currently investigating both 'dimple' $\left(J_{0}\right.$ Bessel) and 3D $(\sin (k x) \times \sin (k y))$ interface perturbations (as opposed to the 2D sinusoidal corrugation in Fig. 3. We are also doing numerical simulations to investigate 2D-3D differences in SN 1987A. Fig. 5 illustrates a simple investigation, comparing the result of imposing 2D sinusoidal, dimple, and $3 \mathrm{D}$ velocity perturbations at the He-H interface after passage of the shock through the interface. The 3D bubble (Fig. $5 \mathrm{c}$ ) grows faster than the $2 \mathrm{D}$ bubble (b), a result already anticipated from previous Nova experiments [19], and the spike grows $\sim 25 \%$ faster in $3 \mathrm{D}$. The dimple spike (b) grows significantly faster than the 2D spike. Fig. 5d shows the distribution by mass of the He velocities at $12,000 \mathrm{~s}$, by which time the blast wave has exited the $\mathrm{H}$ layer. The broadening of the velocity profiles in the He layer (the $\mathrm{H}$ layer is $\sim 20 \% \mathrm{He}$ in the initial model) for the 3D and dimple perturbations is evident.

Work performed under the auspices of the U.S. Department of Energy by the Lawrence Livermore National Laboratory under contract number W-7405-ENG-48. D. A. and J. K. were supported in part by NASA grant NAGW-2450 and NSF grant ASTRO 9015976.

\section{References}

[1] Kane, J., Arnett, D , Remington, B. A., Glendinning, S. G, Castor, J., Wallace, R., Rubenchik, A., Fryxell, B. A , 1997, ApJ 478, L75.

[2] Alon, U., Hecht, J., Mukamel, D , Shvarts, D., 1994, Phys. Rev. Lett., 72, 2867.

[3] Alon, U., Hecht, J , Ofer, D., Shvarts, D., 1995, Phys. Rev Lett., 74, 534

[4] Arnett, D., 1996, Supernovae and Nucleosynthesis, (Princeton: Princeton University Press)

[5] Arnett, D., Bahcall, J. N, Kirshner, R. A., \& Woosley, S. E., 1989, Ann. Rev. A. Ap., 27, 629

[6] Arnett, D., Fryxell, B., Müller, E., 1989, ApJ, 341, L63

[7] Barton, R. T., 1985, In Numerical Astrophysics, ed. J. M. Centrella, J. M. LeBlanc, R. L. Bowers, Boston: Jones and Bartlett Publishors, Inc., pp. 482-497

[8] Blanco, V. M. et al., 1987, ApJ, 320, 589

[9] Budil, K. S., et al., 1996a, Phys. Rev. Lett., 76, 4536

[10] Budil, K. S., et al, 1996b, Rev. Sci. Instrum., 67, 485

[11] Campbell, E. M., Holmes, N. C., Libby, S. B., Remington, B. A., Teller, E., 1996, submitted, Laser and Particle Beams and UCRL-JC-124258 Rev. 2 (1996)

[12] Dionte, G., et al., 1996, Phys. Plasmas, 3, 614

[13] Fryxell, B. A., Müller, E., Arnett, D., 1991, ApJ, 367, 619

[14] Glendinning, S. G., 1992, Rev. Sci. Instrum., 63, 5108

[15] Hanuschik, R. W, Dachs, J., 1987, A\&A, 192, L29

[16] Hecht, J., Alon, U., Shvarts, D., 1994, Phys. Fluids, 6, 4019

[17] Kauffman, R. L., et al., 1994, Phys. Rev. Lett., 73, 2320

[18] Larsen, J. T. and Lane, S. M., 1994, J. Quant. Spect. Rad. Trans. 51, 179

[19] Marinak, M. M., et al., 1995, Phys. Rev. Lett., 75, 3677

[20] McCray, R., 1993, Ann. Rev. A. Ap., 31, 175

[21] Meshkov, E. E., 1969, Izv. Akad. Nauk SSSR Mekh. Zhidk, Gaza 4, 151, [Izv. Acad. Sci. USSR Fluid Dynamics 4, 101 (1969)]

[22] Müller, E., Fryxell, B. A., Arnett, D., 1991, A\&A, 251, 505

[23] Peyser, T. A., et al., 1995, Phys. Rev. Lett., 75, 2332

[24] Lord Rayleigh, 1900, Scientific Papers II, 200, Cambridge, England

[25] Richtmyer, R. D., 1960, Commun. Pure App. Math., 13, 297

[26] Robey H. F , et al., Jan. 1997. Rcv. Sci. Instrum.. in press 
[27] Shvarts, D., private communication.

[28] Shigeyama, T., and Nomoto, K., 1990, ApJ, 360, 212

[29] Taylor, Sir Geoffrey, 1950, Proc. R. Soc. London, A201, 192

[30] Tueller, J., Barthelmy, S., Gehrels, N., Teegarden, B. J., Leventhal, M., MacCallum, C. J, 1990, ApJ, 351, L41

[31] Witteborn, F. C., Bregman, J. D , Wooden, D. H., Pinto, P. A., Rank, D M., Woosley, S. E., Cohen, M., 1989, ApJ, 338, L9

[32] Woodward, P. R. and Colella, P., 1984 J. Comp. Phys., 54, 115 


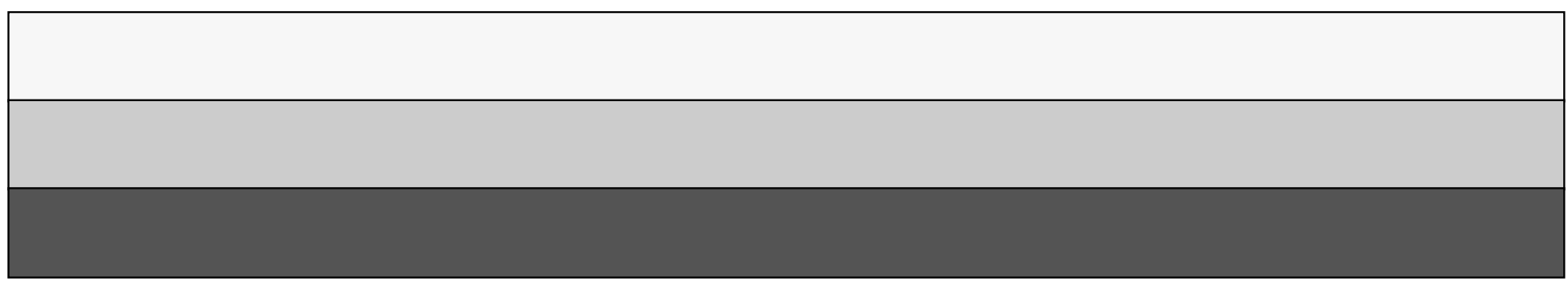

